\title{
O ISOLAMENTO SOCIAL, PELA PANDEMIA DO COVID-19, E A PRÁTICA DE COZINHAR.
}

\section{Viviane Monteiro Dias ${ }^{1 *}$ Juliana Pandini Castelpoggi ${ }^{1}$ Simone Gravitol Marchon ${ }^{1}$ Josiana $^{*}$ Kely Rodrigues Moreira da Silva ${ }^{2}$ José Carlos Siciliano Oliveira ${ }^{3}$ Paula Paraguassú Brandão ${ }^{1}$}

\section{RESUMO}

DIAS, VM.; CASTELPOGGI, JP; MARCHON SG.; SILVA KRM.; OLIVEIRA JCS; BRANDÃO, PP. O isolamento social, pela pandemia do COVID-19, e a prática de cozinhar. Perspectivas Online: Biológicas \& Saúde, v.11 , n. 40 , p. 29 - 38, 2021.

O hábito de cozinhar está desaparecendo de nossas vidas, impulsionados pela globalização e padronização alimentar. A situação situação de isolamento, instituído pelo COVID-19, constitui uma oportunidade para dar origem a um melhor movimento de contracultura, fortalecendo a prática culinária. Este estudo pretende analisar como o isolamento social influenciou no ato de cozinhar das famílias, verificando a adesão ao preparo de refeições, inclusive, correlacionado os sentimentos e afeto envolvidos nesse processo, em meio ao isolamento social promovido pelo COVID- 19. Tratou-se de um estudo transversal, contemplando indivíduos adultos com faixa etária entre 19 e 60 anos, a partir de dados coletados de questionário informatizado utilizando o programa MICROSOFT FORMS. Os indivíduos que aceitaram participar desse estudo assinaram o Termo de Consentimento Livre e Esclarecido, disponível no início do questionário informatizado. Inicialmente, foram respondentes 411 participantes e a partir daí foram aplicados critérios de exclusão para a idade. Posteriormente, foi proposto um subgrupo composto pelos declarantes que realizaram pelo menos 30 dias de isolamento social, consistindo em 264 participantes. Foi identificado que $64,12 \%$ dos participantes possuíam excesso de peso e que o tempo de isolamento não influenciou no prazer em cozinhar. Concluindo, sugere-se que as habilidades culinárias e o resgate pelas preparações feitas em casa ainda representam um desafio a ser superado em tempos de isolamento.

Palavras-chave:Culinária; Antropologia cultural; Isolamento social; Infecções por coronavírus.

\footnotetext{
${ }^{1}$ Instituição Celso Lisboa - Rua Vinte e Quatro de Maio, 797- Engenho Novo, Rio de Janeiro- RJ, Brazil 20950-092, (*) vivimonteirodias@gmail.com;

${ }^{2}$ Universidade do Estado do Pará

3 Universidade Estácio de Sá

Data de recebimento: 26/10/2021.

Aceito para publicação: 06/11/2021.

Data de Publicação 21/12/2021.
}

Persp. Online: biol \& saúde, Campos dos Goytacazes, 40 (11) 29 - 38, 2021

ojs3.perspectivasonline.com.br 


\title{
SOCIAL ISOLATION, THROUGHT THE COVID-19 PANDEMIC AND THE PRACTICE OF COOKING
}

\author{
Viviane Monteiro Dias ${ }^{1 *}$ Juliana Pandini Castelpoggi ${ }^{1}$ Simone Gravitol Marchon ${ }^{1}$ Josiana \\ Kely Rodrigues Moreira da Silva ${ }^{2}$ José Carlos Siciliano Oliveira ${ }^{3}$ Paula Paraguassú \\ Brandão ${ }^{1}$
}

\begin{abstract}
DIAS, VM.; CASTELPOGGI, JP; MARCHON SG.; SILVA KRM.; OLIVEIRA JCS; BRANDÃO, PP. Social isolation, due to the COVID-19 pandemic, and the practice of cooking. Perspectivas Online: Biológicas \& Saúde, v.11 , n. 40 , p. 29 - 38 , 2021.
\end{abstract}

The habit of cooking is disappearing from our lives, driven by globalization and food standardization, this situation of isolation, instituted by COVID-19, constitutes an opportunity to give rise to a better counterculture movement, strengthening the culinary practice. This study aims to analyze how social isolation influenced the cooking of families, verifying adherence to the preparation of meals, including correlating the feelings and affection involved in this process, amid the social isolation promoted by COVID-19. This was a cross-sectional study, covering adult individuals aged between 19 and 60 years, based on data collected from a computerized questionnaire using the MICROSOFT FORMS program.
Individuals who agreed to participate in this study signed the Free and Informed Consent Form, available at the beginning of the computerized questionnaire. Initially, 411 participants were respondents, and from there, exclusion criteria for age were applied. Subsequently, a subgroup was formed consisting of deponents who underwent at least 30 days of social isolation, consisting of 264 participants. It was identified that $64,12 \%$ of the participants were overwheight. And the isolation time did not influence the pleasure of cooking. Completing cooking skills and rescuing home-made preparations still poses a challenge to be overcome in times of isolation.

Keywords: Culinary; Cultural anthropology; Social isolation; Coronavirus infections

\footnotetext{
${ }^{1}$ Instituição Celso Lisboa - Street Vinte e Quatro de Maio, 797- Engenho Novo, Rio de Janeiro- RJ, Brazil 20950-092,

(*) vivimonteirodias@gmail.com;

2 University do Estado do Pará

3 University Estácio de Sá

Receipt: 26/10/2021

Accepted for publication: 06/11/2021.

Date of Publicação 21/12/2021 .
}

Persp. Online: biol \& saúde, Campos dos Goytacazes, 40 (11) 29 - 38, 2021

ojs3.perspectivasonline.com.br 


\section{INTRODUÇÃO}

Desde dezembro 2019, o mundo vem enfrentando uma luta extenuante no combate ao Coronavírus (SARS-CoV-2). Em consonância com as diretrizes internacionais, as medidas de prevenção preconizadas pelo Ministério da Saúde (MS) para minimizar a transmissibilidade da COVID-19 em locais com transmissão comunitária incluem o distanciamento, com isolamento social (BRASIL, 2020; OMS, 2019).

Santos (2020)reafirma "a normalidade da exceção, a atual pandemia não é uma situação de crise claramente contraposta a uma situação de normalidade."

Nesse cenário mundial, houve a necessidade de propor uma discussão sobre o impacto da pandemia e suas implicações no cotidiano das famílias isoladas. Ademais somos seres sociais e culturalmente o hábito de comer em companhia está impregnado em nossa história, assim como a divisão da responsabilidade por encontrar ou adquirir, preparar e cozinhar esses alimentos (BRASIL, 2014a). Ainda que o hábito de cozinhar esteja desaparecendo de nossas vidas, impulsionados pela globalização e padronização alimentar, essa situação de isolamento constitui uma oportunidade para dar origem a um melhor movimento de contracultura, fortalecendo a prática culinária (POLLAN, 2014).

Cozinhar é uma atividade social e cultural, e o ato de cozinhar carrega uma força emocional ou psicológica que expressa a nossa potencialidade humana. Além disso, cozinhar nossas próprias refeições nos faz compreender o significado da saúde, favorece a perpetuação das tradições e dos rituais, promove a autoconfiança e, principalmente, nos permite sentir a suprema satisfação de produzir algo (GALLIAN, 2007).

Como Mauss (2009) bem apontou e se tornou um axioma teórico, a comida é um "fato social complexo". Isso implica que ele cruze todos os aspectos sociais (econômicos, religiosos, jurídicos, políticos etc.), mas também os aspectos básicos, ou seja, fisiológicos e psicológicos, e todos simultaneamente. A comida, para humanos, é um problema que varia da molécula ao símbolo. Mas, em suas ações diárias, a comida é um fenômeno repetido mais de uma vez por dia, é percebida como algo completo e total.

No presente artigo, pretende-se analisar como o isolamento social influenciou no ato de cozinhar das famílias, verificando a adesão ao preparo de refeições, inclusive, correlacionando os sentimentos e afeto envolvidos nesse processo, e identificando os demais hábitos de vida desses indivíduos em meio ao isolamento social promovido pelo COVID- 19.

\section{METODOLOGIA}

Tratou-se de um estudo transversal, contemplando indivíduos adultos com faixa etária entre 19 e 60 anos, a partir de dados coletados de questionário informatizado em plataforma eletrônica (computador, tablet ou celular), utilizando o programa MICROSOFT FORMS, que faz parte do pacote Office 365. A coleta de dados foi online, realizada entre os dias 04 e 24 de junho do ano 2020.

O link foi divulgado pelas redes sociais, através de aplicativos como: Facebook, Instagram, ou Whatsapp, e dessa forma os participantes foram recrutados aleatoriamente,

Persp. Online: biol \& saúde, Campos dos Goytacazes, 40 (11) 29 - 38, 2021

ojs3.perspectivasonline.com.br 
configurando uma amostra não probabilística com viés de conveniência. Os indivíduos que aceitaram participar desse estudo tiveram que assinalar a opção: sim, aceito; referente ao Termo de Consentimento Livre e Esclarecido, disponível no início do questionário informatizado. Todos os participantes da pesquisa foram informados sobre os objetivos da mesma, bem como sobre a segurança em relação a confidencialidade e garantia do anonimato. Por outro lado, foi previamente esclarecido que ao responder o questionário estariam a consentir livremente a utilização da informação por eles prestada para produção de artigos científicos sobre a temática. O projeto de pesquisa foi aprovado pelo Comitê de Ética e Pesquisa do Hospital Naval Marcílio Dias sob o número CAAE 31965120.9.0000.5256.

O questionário foi composto por 35 perguntas fechadas e abertas. As perguntas consistiam em: dados pessoais (e-mail, telefone, sexo, data de nascimento, estado civil); dados sócios econômicos (número de filhos, número de pessoas e de crianças que moram na mesma residência, escolaridade, região nacional de moradia, atividade profissional, renda mensal da família); dados relacionados ao gasto com alimentos, dados antropométricos (peso e estatura), dados sobre o isolamento social, dados relacionados a hábitos saudáveis (atividade física e sua freqüência, sono) e por fim dados relacionados ao hábito de cozinhar (quem realiza as compras, quem cozinha na residência, com que freqüência cozinha e o tempo que se leva para preparar uma refeição, qual tipo de refeição é preparada, qual o prato mais confeccionado, informações sobre os sentimentos relacionado a comida, e o hábito de assistir programas de culinária por diversos meio de comunicação).

A partir do peso e a altura referidos, foi calculado o Índice de Massa Corporal (IMC), por meio da divisão do peso $(\mathrm{kg})$ pela altura $(\mathrm{m})$ ao quadrado, sendo o estado nutricional dos participantes classificado no presente estudo como "peso adequado", quando o IMC esteve entre 18,5 e $24,9 \mathrm{~kg} / \mathrm{m}^{2}$ e "excesso de peso", quando maior e/ou igual a 25 $\mathrm{kg} / \mathrm{m}^{2}(\mathrm{WHO}, 1998)$.

O tratamento estatístico dos dados foi realizado por estatística descritiva, utilizando medidas de tendência central (frequência, média, máximo e mínimo) e medidas de dispersão (desvio padrão), considerando variáveis quantitativas e variáveis categóricas. Foi utilizada estatística inferencial para comparação das variáveis utilizando o teste QuiQuadrado. O nível de significância foi de $p<0,05$. As análises foram feitas utilizando o software Microsoft Excel para Microsoft 365, versão 2019.

\section{RESULTADOS}

Inicialmente, a abordagem estatística foi concentrada na caracterização dos respondentes $(\mathrm{n}=411)$, e a partir daí foram aplicados critérios de exclusão para a idade. Posteriormente, foi criado um subgrupo composto pelos declarantes que realizaram pelo menos 30 dias de isolamento social, consistindo em 264 participantes.

Participaram dessa amostra 227 mulheres (85,9\%), 36 homens $(13,64 \%)$ e 1 pessoa que não caracterizou seu gênero. Observa-se que independentemente da dicotomia sexual, o grupo apresentava elevada variabilidade etária (Coeficiente de Variação > 20,00\%), o que pode ter influenciado o comportamento ao longo da pandemia, dado que as

Persp. Online: biol \& saúde, Campos dos Goytacazes, 40 (11) 29 - 38, 2021

ojs3.perspectivasonline.com.br 
exigências, valores, anseios, estilo de vida, obrigações, compromissos e expectativas são mutáveis no domínio do tempo. A média de idade obervada foi de 39 anos em ambos os sexos.

A Tabela 1 descreve a associação entre a faixa de renda dessas famílias e os tipos de pratos mais confeccionados em suas rotinas. Onde percebeu-se associação positiva entre a renda e o tipo de prato desenvolvido com valor de $\mathrm{p}=0,01 \quad(<0,05)$. Famílias com menores renda estavam preocupadas em pratos saudáveis sem se importar com pratos mais elaborados.

Tabela 1: Correlação da renda familiar salarial das famílias dos respondente e os tipos de pratos confeccionados para alimentação dessas famílias.

\begin{tabular}{|c|c|c|c|c|c|c|c|c|}
\hline & & \multicolumn{6}{|c|}{ Tipos de Pratos } & \multirow[b]{2}{*}{ Total } \\
\hline & $\begin{array}{l}\text { Salários } \\
\text { mínimo }\end{array}$ & $\begin{array}{l}\text { Pratos do } \\
\text { dia-a-dia }\end{array}$ & $\begin{array}{l}\text { Pratos mais } \\
\text { elaborados }\end{array}$ & $\begin{array}{c}\text { Pratos } \\
\text { mais } \\
\text { rápidos }\end{array}$ & $\begin{array}{l}\text { Pratos } \\
\text { regionais }\end{array}$ & $\begin{array}{c}\text { Pratos } \\
\text { saudáveis }\end{array}$ & $\begin{array}{l}\text { Não se } \\
\text { aplica }\end{array}$ & \\
\hline \multirow{5}{*}{ Renda } & $\begin{array}{c}\text { Menos } \\
\text { de } 1\end{array}$ & $0,00 \%$ & $0,00 \%$ & $0,00 \%$ & $0,00 \%$ & $0,76 \%$ & $0,00 \%$ & $1 \%$ \\
\hline & 1 & $2,65 \%$ & $0,00 \%$ & $0,76 \%$ & $0,00 \%$ & $0,00 \%$ & $0,00 \%$ & $3 \%$ \\
\hline & 2 & $3,79 \%$ & $1,52 \%$ & $1,14 \%$ & $0,76 \%$ & $0,76 \%$ & $0,00 \%$ & $8 \%$ \\
\hline & 3 & $3,79 \%$ & $1,14 \%$ & $1,52 \%$ & $0,00 \%$ & $1,52 \%$ & $0,38 \%$ & $8 \%$ \\
\hline & $\begin{array}{l}4 \text { ou } \\
\text { mais }\end{array}$ & $46,97 \%$ & $10,23 \%$ & $9,09 \%$ & $0,00 \%$ & $12,50 \%$ & $0,76 \%$ & $80 \%$ \\
\hline \multicolumn{2}{|c|}{ Total } & $57,20 \%$ & $12,88 \%$ & $12,50 \%$ & $0,76 \%$ & $15,53 \%$ & $1,14 \%$ & $100 \%$ \\
\hline
\end{tabular}

O estudo identificou que $64,12 \%$ dos participantes possuíam excesso de peso. Dentre as mulheres o quantitativo foi de $61,06 \%$, enquanto que os homens conquistaram $83,33 \%$ de ocorrências.

Em relação a estratificação do tempo de isolamento, percebe-se que as pessoas que ficaram isoladas no intervalo de 90-120 dias apresentaram maior prazer em cozinhar em relação aos que relataram isolamento no intervalo de 30-60 dias, porém quando essa hipótese foi testada essa associação expressa um valor de $\mathrm{p}=0,77(>0,05)$, revelando que o tempo de isolamento não influenciou no prazer em cozinhar, conforme expresso na Tabela 2.

Tabela 2: Correlação entre o tempo de isolamento dos respondentes frente ao Covid 19 e o prazer em cozinhar. 


\begin{tabular}{|c|c|c|c|c|c|}
\hline \multicolumn{2}{|c|}{} & \multicolumn{3}{c|}{ Prazer em Cozinhar } & \multirow{2}{*}{ Total } \\
\cline { 3 - 6 } \multicolumn{2}{c|}{} & Sim & Não & Não se aplica & \\
\hline \multirow{3}{*}{$\begin{array}{c}\text { Tempo de } \\
\text { Isolamento }\end{array}$} & $30-90$ & $9,85 \%$ & $1,52 \%$ & $1,14 \%$ & $12,50 \%$ \\
\cline { 2 - 6 } & $120-150$ & $70,83 \%$ & $9,85 \%$ & $5,68 \%$ & $86,36 \%$ \\
\cline { 2 - 6 } & 120 & $0,76 \%$ & $0,38 \%$ & $0,00 \%$ & $1,14 \%$ \\
\hline \multicolumn{2}{|c|}{ Total } & $81,44 \%$ & $11,74 \%$ & $6,82 \%$ & $100,00 \%$ \\
\hline
\end{tabular}

Quanto ao fato de possuir crianças menores de 10 anos morando na mesma coabitação, 110(41,6\%) indivíduos possuíam ao menos 1 criança menor de 10 anos vivendo junto a eles. Sendo o próprio respondente na maioria das vezes a pessoa responsável por cozinhar na sua residência, atingindo um total de $57(21,59 \%)$ pessoas. Onde, $49(18,5 \%)$ respondentes eram os responsáveis por realizar as compras dos alimentos em sua moradia,cozinhando em sua maioria todos os dias 58(21,9\%). Levando de 1 à 2 horas para preparar uma grande refeição, como: almoço e jantar; para grande parte da amostra 59(22,3\%).Houve correlação positiva entre pessoas que possuem crianças em casa com o prazer no ato de cozinhar $(0,18)$.

Quando questionados sobre testar novas receitas e se a audiência em relação a programas de culinária elevou a possibilidade das pessoas acrescentarem novos intens ao seu cardápio, percebeu-se que o tempo de isolamento não teve influência bem como os programas de culinária também não levaram essas mesmas pessoas a reproduzirem suas receitas, conforme demonstrado em associações trabalhadas nas Tabelas 3 e 4 . Com valores de p respectivamente de 0,28 e 1,59.

Tabela 3: Correlação entre o tempo de isolamento e o ato de cozinhar novas receitas.

\begin{tabular}{|c|c|c|c|c|c|}
\cline { 3 - 5 } \multicolumn{2}{c|}{} & \multicolumn{3}{c|}{ Testa Nova receita } & \multirow{2}{*}{ Total } \\
\cline { 2 - 5 } \multicolumn{2}{c|}{} & Sim & Não & Não se aplica & \\
\hline \multirow{3}{*}{$\begin{array}{c}\text { Tempo de } \\
\text { Isolamento }\end{array}$} & $30-90$ & $10,61 \%$ & $1,89 \%$ & $0,00 \%$ & $12,50 \%$ \\
\cline { 2 - 6 } & $90-120$ & $81,44 \%$ & $4,55 \%$ & $0,38 \%$ & $86,36 \%$ \\
\cline { 2 - 6 } & $120-150$ & $1,14 \%$ & $0,00 \%$ & $0,00 \%$ & $1,14 \%$ \\
\hline \multicolumn{2}{|c|}{ Total } & $93,18 \%$ & $6,44 \%$ & $0,38 \%$ & $100,00 \%$ \\
\hline
\end{tabular}

Tabela 4: Correlação entre o tempo assistir programas de culinário e o ato de cozinhar novas receitas.

Persp. Online: biol \& saúde, Campos dos Goytacazes, 40 (11) 29 - 38, 2021

ojs3.perspectivasonline.com.br 


\begin{tabular}{|c|c|c|c|c|c|}
\hline & & \multicolumn{3}{|c|}{ Testa Nova receita } & \multirow{2}{*}{ Total } \\
\hline & & Sim & Não & Não se aplica & \\
\hline \multirow{4}{*}{$\begin{array}{c}\text { Assiste } \\
\text { Programa de } \\
\text { Culinária }\end{array}$} & Assisto mais & $38,64 \%$ & $0,00 \%$ & $0,00 \%$ & $38,64 \%$ \\
\hline & $\begin{array}{l}\text { Mesma } \\
\text { frequência }\end{array}$ & $29,92 \%$ & $0,76 \%$ & $0,38 \%$ & $31,06 \%$ \\
\hline & Não assisto & $21,97 \%$ & $5,30 \%$ & $0,00 \%$ & $27,27 \%$ \\
\hline & Não se aplica & $2,65 \%$ & $0,38 \%$ & $0,00 \%$ & $3,03 \%$ \\
\hline \multicolumn{2}{|c|}{ Total } & $93,18 \%$ & $6,44 \%$ & $0,38 \%$ & $100,00 \%$ \\
\hline
\end{tabular}

\section{DISCUSSÃO}

A pandemia da Covid-19 mudou drasticamente a rotina dos brasileiros. O isolamento social trouxe muitos desafios para as famílias, incluindo a necessidade de elaborarem suas próprias refeições nos domicílios.De acordo com Uggione et al. (2020) cozinhar, para alguns indivíduos, pode ainda ser uma estratégia para reduzir sentimentos como estresse e ansiedade, comuns no contex to da pandemia. Mas a realidade brasileira, no entanto, é marcada por desigualdades de renda, como da forma que populações mais vulneráveis podem não ter essa mesma relação com o ato de cozinhar.

A renda dessas famílias foram afetadas prejudicando o acesso a alimentos em quantidade suficiente e de qualidade. Segundo Silva et al. (2020), há evidências de que a pandemia, apesar de não poder ser responsabilizada integralmente, agravou a situação de insegurança alimentar e nutricional no Brasil. A Lei Orgânica de 2006, garante aos brasileiros o acesso regular e permanente a alimentos de qualidade, de modo que se instaurem práticas alimentares promotoras de saúde. Assim, evidencia-se que a COVID-19 dificulta a promoção de saúde pelas vias alimentares e nutricionais estabelecidas por lei.

Em adição, o presente momento de distanciamento físico pôde ter potencialmente catalisando a adoção do deliveryou take away de comida (BOTELHO; CARDOSO; CANELLA, 2020)com consequente diminuição na prática de cozinhar e piora da relação do consumo de refeições com qualidadealém do aumento no consumo de ultraprocessados, que são formulações industriais feitas inteiramente ou majoritariamente de substâncias extraídas de alimentos.O elevado consumo de alimentos ditos ultraprocessados está intimamente ligado ao amento de sobrepeso e obesidade (BRASIL, 2014b)Um estudo de Andrade et al. (2020), identificou que o consumo de refeições fora de casa favorece a substituição de alimentos tradicionais por alimentos ultraprocessados.

No perfil antropométrico da amostra estudada, entende-se que a maioria apresenta classificação pelo IMC de eutrofia, seguido pela classificação de sobrepeso. Dessa forma padrões inadequados de consumo alimentarpodem estar relacionado com a Pandemia do Covid-19, levando ao alto consumo de alimentos ultraprocessados. No estudo

Persp. Online: biol \& saúde, Campos dos Goytacazes, 40 (11) 29 - 38, 2021

ojs3.perspectivasonline.com.br 
desenvolvido por Lamounier et al. (2021)foram entrevistados 210 profissionais de saúde, onde $45 \%$ da amostra relatou aumento no consumo desse tipo de alimentos.

Em suma a busca por melhorias na qualidade de vida em tempos de COVID-19, gerou inúmeras pesquisas que relatam osmeios descobertos pela população para driblar anseios como:estresse, depressão e doenças mentais que afetam também as formas de apropriação e de alcance dos mais altos níveis de participação nas vivências de lazer cotidiana. A prática de cozinhar pode vir a despertar sentimentos relacionados ao prazere preencher o tempo ocioso de algumas pessoas em isolamento (BICALHO et al., 2021; CLEMENTE; STOPPA, 2020).

Dentre as limitações deste estudo, destacamos a natureza não probabilística da amostra estudada, e o ainda reduzido número absoluto de participantes em algumas macrorregiões do país e nos estratos de menor escolaridade, coleta de dados pela internet, que pode não atingir todos os estratos populacionais, visto que nem todos têm acesso a esse meio de comunicação. A exclusão involuntária dessas pessoas pode ter provocado a sub ou superestimação da proporção dos indicadores. Por fim, destaca-se que o tempo necessário para a realização de uma pesquisa domiciliar representativa em nível nacional, e a taxa de resposta, geralmente baixa,de pesquisas por telefone, faz das pesquisas on-line um método promissor para avaliar e rastrear conhecimentos,comportamentos, estilos de vida e percepções durante surtos de doenças infecciosas em rápida evolução, tais como a COVID-19 (SIDOR; RZYMSKI, 2020). Ademais, o baixo custo relacionado à operacionalização da pesquisa on-line deve ser destacado. Assim, estudos nacionais e internacionais vêm sendo desenvolvidos utilizando métodos de coleta de dados pela internet, utilizando redes de comunicação virtual (ALI et al., 2020).

\section{CONCLUSÕES}

As habilidades culinárias e o resgate pelas preparações feitas em casa ainda representam um desafio a ser superado em tempos de isolamento e emum futuro próximo revelando um cenário pós pandêmico.

Políticas públicas precisam ser desenvolvidas e colocadas em prática para garantia de acesso a alimentos e incentivo a autonomia alimentar dos indivíduos, de forma que os mesmos disfrutem do prazer no ato de confeccionar uma refeição, entendendo todo o seu sistema simbólico e sua representatividade.

\section{REFERÊNCIAS}

ALI, S. H.; FOREMAN, J.; CAPASSO, A.; JONES, A. M.; TOZAN, Y.; DiCLEMENTE, J. R. Social media as a recruitment platform for a nation wide online survey of COVID19 knowledge, beliefs, and practices in the United States: methodology and feasibility analysis. BioMed Central Medical Research Methodology. v.20, n. 116, p.1-12, 2020.

ANDRADE, G. C.; GOMBI-VACA, M. F.; LOUZADA, M. L. C.; AZEREDO, C. M.; LEVY, R. B. The consumption of ultra-processed foods according to eating out occasions. Public Health Nutrition. v.22, n.6, p. 1041-8, 2020.

BICALHO , T.; PASSOS, A. P. P.; MARQUES, A.; SANTOS, C. M. DOS. Impacto da pandemia de COVID-19 nos hábitos de vida da equipe de enfermagem atuante em

Persp. Online: biol \& saúde, Campos dos Goytacazes, 40 (11) 29 - 38, 2021

ojs3.perspectivasonline.com.br 
unidades de terapia intensiva de hospitais do município de Campos dos Goytacazes-RJ. Biológicas \& Saúde, v. 11, n. 38, p. 15-16, 9 set. 2021.

BOTElHO, L. V.; CARDOSO, L. O.; CANELlA, D. S. COVID-19 e ambiente alimentar digital no Brasil: reflexões sobre a influência da pandemia no uso de aplicativos de delivery de comida. Cadernos de Saúde Pública [online].v. 36, n. 11, 2020.

BRASIL, Ministério da Saúde, Brasil. Guia alimentar para a população brasileira. $2^{\text {a }}$ Edição.Brasília, 2014a.

BRASIL, Ministério da Saúde, Secretaria de Atenção Primária à Saúde. Protocolo de manejo clínico do coronavírus (COVID-19) na atenção primária à saúde. Março de 2020. Disponível em: https://coronavirus.saude.gov.br/manejo-clinico-e-tratamento. Acesso em: 10out. 2020.

BRASIL, Ministério da Saúde. Guia Alimentar para a População Brasileira, $2^{\mathrm{a}}$ ed, 2014b.

CLEMENTE, A. C. F.; STOPPA, E. A. Lazer doméstico em tempos de pandemia da Covid. Revista do Programa de Pós-graduação Interdisciplinar em Estudos do Lazer. v.23, n. 3, 2020.

GALLIAN, D. M. C. A desumanização do comer. Estudos avançados. v.21, n. 60, p. 179-184, 2007.

LAMOUNIER, A.M.; FRÕES, B.T.G.; BARBOSA, L.M.R.; OLIVEIRA, L.F.; TORRES, M.F.; MACEDO, M.T.S.; ROCHA, J.S.B. Consumo de alimentos ultraprocessados em profissionais de saúde durante o COVID-19. Perspectivas Online: Biológicas \& Saúde, v.11 , n.37, p.62-70, 2021.

MAUSS, M. Ensayo sobre eldon: Forma yfuncióndel intercambio en lãs sociedades arcaicas. Buenos Aires: Katz editores, 2009.

OMS, Organização Mundial de Saúde. World Health Organization.Coronavirus disease (COVID-19) pandemic. Disponível em: https://www.who.int/emergencies/diseases/novel-coronavirus-2019. Acesso em 10 out. 2020.

POLLAN, M. Cozinhar: uma história natural da transformação. $1^{a}$ edição.Rio de Janeiro: Intrínseca, 2014.

SANTOS, B. S. A Cruel Pedagogia do Vírus. Editora Almedina. Biblioteca Nacional de Portugal, 2020.

SIDOR, A.; RZYMSKI, P. Dietary choices and habits during COVID-19 lockdown: experience from Poland. Nutrients. v.12, n. 6, p.1657, 2020.

SILVA, R.C.R.; PEREIRA, M.; CAMPELLO, T.; ARAGÃO, E.; FERREIRA, A.J.F.; BARRETO, M.L.; SANTOS, S.M.C. Implicações da pandemia COVID-19 para a segurança alimentar e nutricional no Brasil. Ciência \& Saúde Coletiva, v.25, n.7, p.2504-2504, 2020.

Persp. Online: biol \& saúde, Campos dos Goytacazes, 40 (11) 29 - 38, 2021

ojs3.perspectivasonline.com.br 
UGGIONI, P. L.; FERNANDES, C. M.; GERALDO, A. P . G.; FERNANDES, A. C.; MAZZONETTO, A. C.; BERNARDO, G. L. Habilidades culinárias em tempos de pandemia pela Covid-19. Revista de Nutrição. v.33, e200172, 2020.

WHO, World Health Organization. Obesity: preventing and managing the global epidemic. Report of a WHO consultation on obesity. Geneva; 1998. 